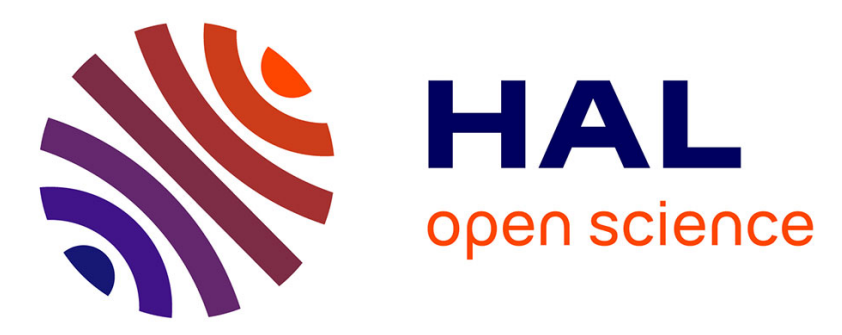

\title{
Espaces de Hardy et compacité par compensation : un résultat en dimension deux
}

\author{
Fabien Flori, Catherine Giacomoni
}

\section{To cite this version:}

Fabien Flori, Catherine Giacomoni. Espaces de Hardy et compacité par compensation : un résultat en dimension deux. 2010. hal-00604057

\section{HAL Id: hal-00604057 \\ https://hal.science/hal-00604057}

Preprint submitted on 30 Jun 2011

HAL is a multi-disciplinary open access archive for the deposit and dissemination of scientific research documents, whether they are published or not. The documents may come from teaching and research institutions in France or abroad, or from public or private research centers.
L'archive ouverte pluridisciplinaire HAL, est destinée au dépôt et à la diffusion de documents scientifiques de niveau recherche, publiés ou non, émanant des établissements d'enseignement et de recherche français ou étrangers, des laboratoires publics ou privés. 


\title{
ESPACES DE HARDY ET COMPACITÉ PAR COMPENSATION \\ Un RÉSULTAT EN DIMENSION 2
}

\author{
Fabien Flori \\ Université Française d'Egypte, fabien.flori@ufe.edu.eg \\ Catherine Giacomoni \\ Université de Corse, giaco@univ-corse.fr
}

Résumé - Nous donnons un résultat faisant apparaître un phénomène de léger gain en régularité dans des quantités non linéaires par rapport à leur régularité apparente.

On définit l'espace de Hardy généralisé introduit par E. Stein et G. Weiss [3] que nous noterons $\mathcal{H}^{1}$

$$
\mathcal{H}^{1}\left(\mathbb{R}^{2}\right)=\left\{f \in L^{1}\left(\mathbb{R}^{2}\right) / \sup _{\eta \geq 0}\left|h_{\eta} \star f\right| \in L^{1}\left(\mathbb{R}^{2}\right)\right\}
$$

où $h_{\eta}(x)=\frac{1}{\eta^{2}} h\left(\frac{x}{\eta}\right) \geq 0$ appartient à $C_{0}^{\infty}\left(\mathbb{R}^{2}\right)$ et vérifie :

$$
\operatorname{supp}_{\eta}(x) \subset B_{\eta}^{x}, \quad \int_{\mathbb{R}^{2}} h_{\eta}(x) d x=1
$$

On travaille dans ce qui suit sur des espaces de Hardy définis sur des domaines bornés et en particulier on pose :

$$
\mathcal{H}_{z}^{1}(\Omega)=\left\{f \in L^{1}(\Omega) / f_{z} \in \mathcal{H}^{1}\left(\mathbb{R}^{2}\right)\right\}
$$

où $f_{z}$ est le prolongement de $f$ par 0 dans $\mathbb{R}^{2}$. Notons que toute fonction $f$ dans $\mathcal{H}_{z}^{1}(\Omega)$ vérifie $\int_{\Omega} f d x=0$. Une norme sur cet espace est donnée par

$$
\|f\|_{\mathcal{H}_{z}^{1}(\Omega)}=\left\|f_{z}\right\|_{\mathcal{H}_{z}^{1}\left(\mathbb{R}^{2}\right)} .
$$

On définit l'espace de Hardy-Sobolev $W^{1} \mathcal{H}_{z}^{1}(\Omega)$ comme l'ensemble des fonctions $\phi$ de $\mathcal{H}_{z}^{1}(\Omega)$ dont les dérivées $\frac{\partial \Phi}{\partial x_{i}}$ appartiennent aussi à $\mathcal{H}_{z}^{1}(\Omega)$. On note $W_{0}^{1} \mathcal{H}_{z}^{1}(\Omega)$ la fermeture de $C_{0}^{\infty}(\Omega)$ dans $W^{1} \mathcal{H}_{z}^{1}(\Omega)$. On munit $W_{0}^{1} \mathcal{H}_{z}^{1}(\Omega)$ de la norme :

$$
\|\phi\|_{W_{0}^{1} \mathcal{H}_{z}^{1}(\Omega)}=\|\operatorname{div} \phi\|_{\mathcal{H}_{z}^{1}(\Omega)}+\|\operatorname{curl} \phi\|_{\mathcal{H}_{z}^{1}(\Omega)} .
$$

On montre le résultat suivant :

Théorème 1 - Soit $\Omega$ un ouvert borné de $\mathbb{R}^{2}$ et $v=\left(v_{1}, v_{2}\right) \in L^{\infty}\left(0, T ; L^{2}(\Omega)\right) \cap$ $L^{2}\left(0, T ; H_{0}^{1}(\Omega)\right)$ alors

$$
v_{i} v_{j} \in L^{2}\left(0, T ; W^{1} \mathcal{H}_{z}^{1}(\Omega)\right) \quad \forall i, j \in\{1,2\} .
$$

Remarque 1 - On retrouve ainsi un résultat classique en mécanique des fluides lorsque le fluide est incompressible et que $\operatorname{div} v=0$. En effet, si $v_{i} v_{j} \in$ 
$L^{2}\left(0, T ; W^{1} \mathcal{H}_{z}^{1}(\Omega)\right)$ alors le terme d'advection $(v . \nabla) v$ appartient à $L^{2}\left(0, T ; \mathcal{H}_{z}^{1}(\Omega)\right)$ avec $\mathcal{H}_{z}^{1}(\Omega) \subset H^{-1}(\Omega)$ dans le cas de la dimension $2[2]$.

Lemme 1 - Si $v \in L^{\infty}\left(0, T ; L^{2}(\Omega)\right) \cap L^{2}\left(0, T ; H_{0}^{1}(\Omega)\right)$ alors :

$$
\operatorname{curl}\left(v_{j} v\right) \in L^{2}\left(0, T ; \mathcal{H}_{z}^{1}(\Omega)\right) \quad \forall j \in\{1,2\} .
$$

Preuve du lemme 1 - Dans ce qui suit, comme $v \in L^{\infty}\left(0, T ; L^{2}(\Omega)\right) \cap$ $L^{2}\left(0, T ; H_{0}^{1}(\Omega)\right)$, on prolonge $v$ et $\frac{\partial v_{j}}{\partial x_{i}}$ par 0 sur $\mathbb{R}^{2}-\Omega$.

Pour tout $x \in \Omega$, on note $B_{\eta}^{x}$ la boule de $\mathbb{R}^{2}$ de centre $x$ et de rayon $\eta$. Sur chaque boule $B_{\eta}^{x}$, on peut toujours décomposer $v$ de façon unique sous la forme :

$$
v=\nabla p_{\eta}^{x}+\operatorname{Curl} q_{\eta}^{x}
$$

avec Curlq $=\left(\frac{\partial q}{\partial x_{2}},-\frac{\partial q}{\partial x_{1}}\right), p_{\eta}^{x} \in H^{1}\left(B_{\eta}^{x}\right)$ et $q_{\eta}^{x} \in H_{0}^{1}\left(B_{\eta}^{x}\right)$. Bien sûr, si $B_{\eta}^{x}$ et $B_{\eta^{\prime}}^{x^{\prime}}$ sont deux boules telles que $B_{\cap}=B_{\eta}^{x} \cap B_{\eta^{\prime}}^{x^{\prime}} \neq \emptyset$, sur chacune de ces boules, $v$ se décompose de façon unique et il est clair que l'on n'a pas nécessairement $\operatorname{Curl} q_{\eta}^{x}=\operatorname{Curl} q_{\eta^{\prime}}^{x^{\prime}}$ sur $B_{\cap}$. Toutefois, on a curl $u=\operatorname{curl} \operatorname{Curl} q_{\eta}^{x}=\operatorname{curl} \operatorname{Curl} q_{\eta^{\prime}}^{x^{\prime}}$ sur $B_{\cap}$. Cette propriété sera utile par la suite.

Sur chaque boule $B_{\eta}^{x}$ on a la décomposition :

$$
\operatorname{curl}\left(v_{j} v\right)=\operatorname{curl}\left(v_{j} \operatorname{Curl} q_{\eta}^{x}\right)-\nabla p_{\eta}^{x} \operatorname{Curl} v_{j} .
$$

On estime séparément chacun des termes du second membre en utilisant les propriétés de la décomposition $v=\nabla p_{\eta}^{x}+\operatorname{Curl} q_{\eta}^{x}$ sur chaque boule. On obtient une borne de $\operatorname{curl}\left(v_{j} v\right)$ dans $L^{2}\left(0, T ; \mathcal{H}_{z}^{1}(\Omega)\right)$ indépendante de $\eta$.

1ère étape - Estimation de $\nabla p_{\eta}^{x} \operatorname{Curl} v_{j}$.

On adapte le théorème div - curl [1] au terme $\nabla p_{\eta}^{x} \operatorname{Curl} v_{j}$.

$$
\left|\nabla p_{\eta}^{x} \operatorname{Curl} v_{j} * h_{\eta}(x)\right|=\left|\int_{B_{\eta}^{x}}\left(\nabla p_{\eta}^{x} \operatorname{Curl} v_{j}\right)(y) \frac{1}{\eta^{2}} h\left(\frac{x-y}{\eta}\right) d y\right|
$$

Comme $h_{\eta}(x)$ est à support compact sur $B_{\eta}^{x}$, il vient :

$$
\left|\nabla p_{\eta}^{x} \operatorname{Curl} v_{j} * h_{\eta}(x)\right|=\left|\int_{B_{\eta}^{x}} \nabla p_{\eta}^{x}(y)\left(\frac{v_{j}-\overline{v_{j}}}{\eta}\right)(y) \frac{1}{\eta^{2}} \operatorname{Curl} h\left(\frac{x-y}{\eta}\right) d y\right|
$$

où $\overline{v_{j}}=f_{B_{\eta}^{x}} v_{j} d y$ et $\nabla p_{\eta}^{x}=v-\operatorname{Curl} q_{\eta}^{x}$. Si on pose $C_{0}=\pi\|\operatorname{Curl} h\|_{\infty}$ et $\operatorname{mes}\left(B_{\eta}^{x}\right)=\pi \eta^{2}$ on obtient :

$$
\left|\nabla p_{\eta}^{x} \operatorname{Curl} v_{j} * h_{\eta}(x)\right| \leq C_{0} f_{B_{\eta}^{x}}\left|\frac{v_{j}(y)-\overline{v_{j}}}{\eta}\right||v(y)| d y+C_{0} f_{B_{\eta}^{x}}\left|v_{j}(y)-\overline{v_{j}}\right|\left|\frac{\operatorname{Curl} q_{\eta}^{x}(y)}{\eta}\right| d y
$$

Avec l'inégalité de Holder, en posant $\frac{1}{\beta}+\frac{1}{\beta^{\prime}}=1$, on a :

$$
\left|\nabla p_{\eta}^{x} \operatorname{Curl} v_{j} * h_{\eta}(x)\right| \leq C_{1}\left(f_{B_{\eta}^{x}}\left|\frac{v_{j}(y)-\overline{v_{j}}}{\eta}\right|^{\beta^{\prime}} d y\right)^{\frac{1}{\beta^{\prime}}}\left(f_{B_{\eta}^{x}}|v|^{\beta} d y\right)^{\frac{1}{\beta}}
$$




$$
+C_{1}\left(f_{B_{\eta}^{x}}\left|v_{j}(y)-\overline{v_{j}}\right|^{\beta} d y\right)^{\frac{1}{\beta}}\left(f_{B_{\eta}^{x}}\left|\frac{\operatorname{Curl} q_{\eta}^{x}}{\eta}\right|^{\beta^{\prime}} d y\right)^{\frac{1}{\beta^{\prime}}}
$$

On applique ensuite l'inégalité de Sobolev-Poincaré avec $\frac{1}{\alpha}-\frac{1}{2}=1-\frac{1}{\beta}=\frac{1}{\beta^{\prime}}$. Il vient d'une part :

$$
\left(f_{B_{\eta}^{x}}\left|\frac{v_{j}(y)-\overline{v_{j}}}{\eta}\right|^{\beta^{\prime}} d y\right)^{\frac{1}{\beta^{\prime}}} \leq C_{2}\left(f_{B_{\eta}^{x}}\left|\nabla v_{j}\right|^{\alpha} d y\right)^{\frac{1}{\alpha}}
$$

et d'autre part, comme $\int_{B_{\eta}^{x}} \operatorname{Curl} q_{\eta}^{x} d y=0, \operatorname{Curl} q_{\eta}^{x} \cdot n=0$ sur $\partial B_{\eta}^{x}$ et $\operatorname{curl} v=$ $\operatorname{curl} \operatorname{Curl} q_{\eta}^{x}$ sur $B_{\eta}^{x}$, il est clair avec l'inégalité de Sobolev-Poincaré que :

$$
\left(f_{B_{\eta}^{x}}\left|\frac{\operatorname{Curl} q_{\eta}^{x}}{\eta}\right|^{\beta^{\prime}} d y\right)^{\frac{1}{\beta^{\prime}}} \leq C_{3}\left(f_{B_{\eta}^{x}}|\operatorname{curl} v|^{\alpha} d y\right)^{\frac{1}{\alpha}}
$$

Ainsi :

$$
\begin{gathered}
\sup _{\eta>0}\left|\nabla p_{\eta}^{x} \operatorname{Curl} v_{j} * h_{\eta}(x)\right| \leq C_{4}\left(\sup _{\eta>0} \int_{B_{\eta}^{x}}\left|\nabla v_{j}\right|^{\alpha} d y\right)^{\frac{1}{\alpha}}\left(\sup _{\eta>0} \int_{B_{\eta}^{x}}|v|^{\beta} d y\right)^{\frac{1}{\beta}} \\
+C_{5}\left(\int_{B_{\eta}^{x}}\left|v_{j}(y)-\overline{v_{j}}\right|^{\beta} d y\right)^{\frac{1}{\beta}}\left(\sup _{\eta>0} \int_{B_{\eta}^{x}}|\operatorname{curl} v|^{\alpha} d y\right)^{\frac{1}{\alpha}}
\end{gathered}
$$

avec

$$
\left(f_{B_{\eta}^{x}}\left|v_{j}(y)-\bar{v}_{j}\right|^{\beta} d y\right)^{\frac{1}{\beta}} \leq C_{5}\left(f_{B_{\eta}^{x}}\left|v_{j}(y)\right|^{\beta} d y\right)^{\frac{1}{\beta}}+C_{5} f_{B_{\eta}^{x}}\left|v_{j}(y)\right| d y
$$

En posant $\sup _{\eta>0} f_{B_{\eta}^{x}}|f| d y=M(f)$, on parvient à :

$$
\begin{gathered}
\int_{\Omega} \sup _{\eta>0}\left|\nabla p_{\eta}^{x} \operatorname{Curl} v_{j} * h_{\eta}(x)\right| d x \leq C_{4} \int_{\Omega}\left(M\left(\left|\nabla v_{j}\right|^{\alpha}\right)\right)^{\frac{1}{\alpha}}\left(M\left(|v|^{\beta}\right)\right)^{\frac{1}{\beta}} d x \\
+C_{5} \int_{\Omega}\left(M\left(\left|v_{j}\right|^{\beta}\right)\right)^{\frac{1}{\beta}}\left(M\left(|\operatorname{curl} v|^{\alpha}\right)\right)^{\frac{1}{\alpha}} d x+C_{5} \int_{\Omega}\left(M\left(\left|v_{j}\right|\right)\right)\left(M\left(|\operatorname{curl} v|^{\alpha}\right)\right)^{\frac{1}{\alpha}} d x
\end{gathered}
$$

On utilise à nouveau l'inégalité de Holder $\frac{1}{p}+\frac{1}{p^{\prime}}=1$ :

$$
\begin{gathered}
\int_{\Omega} \sup _{\eta>0}\left|\nabla p_{\eta}^{x} \operatorname{Curl} v_{j} * h_{\eta}(x)\right| d x \leq C_{6}|| M\left(\left|\nabla v_{j}\right|^{\alpha}\right)\left\|_{L^{\frac{p}{\alpha}}(\Omega)}^{\frac{1}{\alpha}}\right\| M\left(|v|^{\beta}\right) \|_{L^{\frac{p^{\prime}}{\beta}}(\Omega)}^{\frac{1}{\beta}} \\
+C_{7}\left\|M\left(\left|v_{j}\right|^{\beta}\right)\right\|_{L^{\frac{p^{\prime}}{\beta}(\Omega)}}^{\frac{1}{\beta}}\left\|M\left(|\operatorname{curl} v|^{\alpha}\right)\right\|_{L^{\frac{p}{\alpha}}(\Omega)}^{\frac{1}{\alpha}} \\
+C_{8}\left\|M\left(\left|v_{j}\right|\right)\right\|_{L^{p^{\prime}}(\Omega)}\left\|M\left(|\operatorname{curl} v|^{\alpha}\right)\right\|_{L^{\frac{p}{\alpha}}(\Omega)}^{\frac{1}{\alpha}} .
\end{gathered}
$$


Finalement en choisissant $\beta^{\prime}<p^{\prime}$ et $\alpha<p$ on peut appliquer le théorème maximal de Hardy-Littlewood :

$\int_{\Omega} \sup _{\eta>0}\left|\nabla p_{\eta}^{x} \operatorname{Curl} v_{j} * h_{\eta}(x)\right| d x \leq C_{9}\left\|\nabla v_{j}\right\|_{L^{p}(\Omega)}\|v\|_{L^{p^{\prime}}(\Omega)}+C_{10}\left\|v_{j}\right\|_{L^{p^{\prime}}(\Omega)}\|\operatorname{curl} v\|_{L^{p}(\Omega)}$

Si on fixe $p=p^{\prime}=2$, comme $v \in L^{\infty}\left(0, T ; L^{2}(\Omega)\right) \cap L^{2}\left(0, T ; H_{0}^{1}[\Omega)\right)$, alors :

$$
\int_{\Omega} \sup _{\eta>0}\left|\nabla p_{\eta}^{x} \operatorname{Curl} v_{j} * h_{\eta}(x)\right| d x \leq C(t) \in L^{2}(0, T)
$$

Finalement, comme d'une part $\int_{\Omega} \nabla p_{\eta}^{x} \operatorname{Curl} v_{j} d x=0$ et que d'autre part $\nabla p_{\eta}^{x} \operatorname{Curl} v_{j}=$ 0 sur $\mathbb{R}^{2}-\Omega$ (car on a prolongé $v_{j}$ et $\frac{\partial v_{j}}{\partial x_{i}}$ par 0 sur $\mathbb{R}^{2}-\Omega$ ), on obtient:

$$
\nabla p_{\eta}^{x} \operatorname{Curl} v_{j} \in L^{2}\left(0, T ; \mathcal{H}_{z}^{1}(\Omega)\right)
$$

2ème étape - Estimation de $\operatorname{curl}\left(v_{j} \operatorname{Curl} q_{\eta}^{x}\right)$.

On a :

$$
\left|\operatorname{curl}\left(v_{j} \operatorname{Curl} q_{\eta}^{x}\right) * h_{\eta}(x)\right|=\left|\int_{B_{\eta}^{x}} \frac{1}{\eta^{2}} \operatorname{Curl} h\left(\frac{x-y}{\eta}\right) v_{j}(y) \frac{\operatorname{Curl} q_{\eta}^{x}(y)}{\eta} d y\right|
$$

et avec l'inégalité de Holder si $\frac{1}{\beta}+\frac{1}{\beta^{\prime}}=1$ :

$$
\left|\operatorname{curl}\left(v_{j} \operatorname{Curl} q_{\eta}^{x}\right) * h_{\eta}(x)\right| \leq C_{1}\left|\left(f_{B_{\eta}^{x}}\left|v_{j}\right|^{\beta}\right)^{\frac{1}{\beta}}\left(f_{B_{\eta}^{x}}\left(\frac{\left|\operatorname{Curl} q_{\eta}^{x}\right|}{\eta}\right)^{\beta^{\prime}}\right)^{\frac{1}{\beta^{\prime}}}\right|
$$

Comme $f_{B_{\eta}^{x}} \operatorname{Curl} q_{\eta}^{x} d y=0, \operatorname{Curl} q_{\eta}^{x} \cdot n=0$ sur $\partial B_{\eta}^{x}$ et $\operatorname{curl} v=\operatorname{curl} \operatorname{Curl} q_{\eta}^{x} \operatorname{sur} B_{\eta}^{x}$, il est clair avec l'inégalité de Sobolev-Poincaré que :

$$
\left(f_{B_{\eta}^{x}}\left|\frac{\operatorname{Curl} q_{\eta}^{x}}{\eta}\right|^{\beta^{\prime}} d y\right)^{\frac{1}{\beta^{\prime}}} \leq C_{2}\left(f_{B_{\eta}^{x}}|\operatorname{curl} v|^{\alpha} d y\right)^{\frac{1}{\alpha}}
$$

avec $\frac{1}{\alpha}-\frac{1}{2}=1-\frac{1}{\beta}=\frac{1}{\beta^{\prime}}$. Ainsi, en posant $\sup _{\eta>0} \int_{B_{\eta}^{x}}|f|=M(f)$, on parvient à :

$$
\int_{\Omega} \sup _{\eta>0}\left|\operatorname{curl}\left(v_{j} \operatorname{Curl} q_{\eta}^{x}\right) * h_{\eta}(x)\right| d x \leq C_{4} \int_{\Omega}\left(M\left(\left|v_{j}\right|^{\beta}\right)\right)^{\frac{1}{\beta}}\left(M\left(|\operatorname{curl} v|^{\alpha}\right)\right)^{\frac{1}{\alpha}} d x
$$

et toujours avec l'inégalité de Holder si $\frac{1}{p}+\frac{1}{p^{\prime}}=1$ :

$$
\int_{\Omega} \sup _{\eta>0}\left|\operatorname{curl}\left(v_{j} \operatorname{Curl} q_{\eta}^{x}\right) * h_{\eta}(x)\right| d x \leq C_{5}\left\|M\left(\left|v_{j}\right|^{\beta}\right)\right\|_{L^{\frac{p}{\beta}(\Omega)}}^{\frac{1}{\beta}} \|\left. M\left(|\operatorname{curl} v|^{\alpha}\right)\right|_{L^{\frac{p^{\prime}}{\alpha}(\Omega)}} ^{\frac{1}{\alpha}} .
$$


Finalement, si on choisit $\beta<p=2$ et $\alpha<p^{\prime}=2$, le théorème du maximum de Hardy-Littlewood montre que :

$$
\int_{\Omega} \sup _{\eta>0}\left|\operatorname{curl}\left(v_{j} \operatorname{Curl} q_{\eta}^{x}\right) * h_{\eta}(x)\right| d x \leq C_{6}\left\|v_{j}\right\|_{L^{2}(\Omega)}\|\operatorname{curl} v\|_{L^{2}(\Omega)} \in L^{2}(0, T) .
$$

Finalement, comme d'une part :

$$
\int_{\Omega} \operatorname{curl}\left(v_{j} \operatorname{Curl} q_{\eta}^{x}\right) d x=0
$$

et que d'autre part $\operatorname{curl}\left(v_{j} \operatorname{Curl} q_{\eta}^{x}\right)=0$ sur $\mathbb{R}^{2}-\Omega$ (car on a prolongé $v_{j}$ et $\frac{\partial v_{j}}{\partial x_{i}}$ par 0 sur $\mathbb{R}^{2}-\Omega$ ), on obtient:

$$
\operatorname{curl}\left(v_{j} \operatorname{Curl} q_{\eta}^{x}\right) \in L^{2}\left(0, T ; \mathcal{H}_{z}^{1}(\Omega)\right) .
$$

De $(0.1)$ et $(0.2)$ on tire le résultat annoncé dans le lemme.

Lemme 2 - Si $v \in L^{\infty}\left(0, T ; L^{2}(\Omega)\right) \cap L^{2}\left(0, T ; H_{0}^{1}(\Omega)\right)$ alors :

$$
\operatorname{div}\left(v_{j} v\right) \in L^{2}\left(0, T ; \mathcal{H}_{z}^{1}(\Omega)\right) .
$$

Preuve du lemme 2 - En dimension 2 on remarque que :

$$
\operatorname{div}\left(v_{j} v\right)=-\operatorname{curl}\left(v_{j} \alpha(v)\right), \quad \alpha(v)=\left(-v_{2}, v_{1}\right) .
$$

Sur chaque boule $B_{\eta}^{x}$, on peut toujours décomposer $\alpha(v)$ de façon unique sous la forme :

$$
\alpha(v)=\nabla r_{\eta}^{x}+\operatorname{Curl} s_{\eta}^{x}
$$

avec $r_{\eta}^{x} \in H^{1}\left(B_{\eta}^{x}\right)$ et $s_{\eta}^{x} \in H_{0}^{1}\left(B_{\eta}^{x}\right)$. On remarque que :

$$
-\operatorname{div}\left(v_{j} v\right)=\operatorname{curl}\left(v_{j} \alpha(v)\right)=\operatorname{curl}\left(v_{j} \operatorname{Curl} s_{\eta}^{x}\right)-\operatorname{Curl} v_{j} \nabla r_{\eta}^{x} .
$$

On procède alors comme au lemme 1 et on prouve que :

$$
\begin{gathered}
\int_{\Omega} \sup _{\eta>0}\left|\operatorname{Curl} v_{j} \nabla r_{\eta}^{x} * h_{\eta}(x)\right| d x \leq C_{1}\left\|\nabla v_{j}\right\|_{L^{2}(\Omega)}\|\alpha(v)\|_{L^{2}(\Omega)} \\
+C_{2}\left\|v_{j}\right\|_{L^{2}(\Omega)}\|\operatorname{curl} \alpha(v)\|_{L^{2}(\Omega)} \in L^{2}(0, T),
\end{gathered}
$$

et

$$
\int_{\Omega} \sup _{\eta>0}\left|\operatorname{curl}\left(v_{j} \operatorname{Curl} s_{\eta}^{x}\right) * h_{\eta}(x)\right| d x \leq C_{3}\left\|u_{j}\right\|_{L^{2}(\Omega)}\|\operatorname{curl} \alpha(u)\|_{L^{2}(\Omega)} \in L^{2}(0, T)
$$

d'où l'on déduit le résultat annoncé.

Preuve du théorème 1 - La preuve du théorème est une simple conséquence des lemmes 1 et 2 .

\section{RÉFÉRENCES}

[1] R. Coifman, P.L. Lions, Y. Meyer, S. Semmes : Séminaire Equations aux Dérivées Partielles - Ecole Polytechnique 14, (1989-1990), p. 1-8.

[2] J. Hogan, C. LI, A. McIntosh, K. Zhang : Ann. Inst. Henri Poincaré 17, (2000), p. 193-217.

[3] E. Stein et G. Weiss : Acta Math., 103 (1960), p. 25-62. 